\title{
Patterns of dietary behaviours identified by latent class analysis are associated with chronic uninvestigated dyspepsia
}

\author{
Ammar Hassanzadeh Keshteli ${ }^{1,2}$, Awat Feizi ${ }^{3}$, Ahmad Esmaillzadeh $^{4,5 *}$, Fatemeh Zaribaf ${ }^{5}$, \\ Christine Feinle-Bisset ${ }^{6}+$, Nicholas J. Talley ${ }^{7,8}$ and Peyman Adibi ${ }^{1}$ \\ ${ }^{1}$ Integrative Functional Gastroenterology Research Center, Isfahan University of Medical Sciences, Isfahan, Iran \\ ${ }^{2}$ Department of Medicine, University of Alberta, Edmonton, AB, Canada \\ ${ }^{3}$ Department of Epidemiology and Biostatistics, School of Public Health, Isfahan University of Medical Sciences, Isfahan, Iran \\ ${ }^{4}$ Food Security Research Center, Isfahan University of Medical Sciences, Isfahan, Iran \\ ${ }^{5}$ Department of Community Nutrition, School of Nutrition and Food Science, Isfahan University of Medical Sciences, \\ PO Box 81745-151, Isfahan, Iran \\ ${ }^{6}$ Discipline of Medicine, University of Adelaide, Adelaide, SA, Australia \\ ${ }^{7}$ Division of Gastroenterology and Hepatology, Mayo Clinic, Rochester, MN, USA \\ ${ }^{8}$ Faculty of Health, University of Newcastle, Callaghan, NSW, 2308, Australia
}

(Submitted 13 June 2013 - Final revision received 3 May 2014 - Accepted 9 November 2014 - First published online 17 February 2015)

\section{Abstract}

To our knowledge, no study has assessed the relationships between patterns of dietary behaviours, identified by latent class analysis (LCA), and chronic uninvestigated dyspepsia (CUD). The present study was conducted to determine the association between the patterns of dietary behaviours, identified by LCA, and CUD in a large sample of adults. In a cross-sectional study conducted on 4763 Iranian adults, we assessed the patterns of dietary behaviours in four domains, including 'meal patterns', 'eating rate', 'intra-meal fluid intake' and 'meal-to-sleep interval', as identified by LCA, using a pre-tested comprehensive questionnaire. Patients with CUD were identified using the Rome III diagnostic criteria. CUD was prevalent in $15 \cdot 2 \%(95 \%$ CI $14 \cdot 4,16 \cdot 2 \% ; n$ 723) of patients. Early satiation occurred in $6 \cdot 3 \%(n 302)$ of patients, bothersome postprandial fullness in $8.0 \%(n 384)$ of patients and epigastric pain in 7.8\% $(n 371)$ of patients. We defined two distinct classes of meal patterns: 'regular' and 'irregular'. For eating rates, three classes were defined: 'moderate', 'moderate-to-slow' and 'moderate-to-fast'. Participants were identified as ingesting fluid with meals in two major classes: 'moderate intra-meal drinking' and 'high intra-meal drinking'. In terms of the interval between meals and sleeping, two distinct classes were identified: 'short meal-to-sleep interval' and 'long meal-to-sleep interval'. After controlling for potential confounders, the 'irregular meal pattern' was significantly associated with a greater odds of CUD (OR 1·42, 95\% CI 1·12, 1.78) compared with a 'regular meal pattern'. Individuals with a 'moderate-to-fast eating rate' were more likely to have CUD compared with those who had a 'moderate eating rate' (OR 1.42, $95 \%$ CI 1·15, 1.75). Patterns of the 'meal-to-sleep interval' and 'intra-meal fluid intake' were not significantly associated with CUD. In conclusion, the 'irregular meal pattern' and the 'moderate-to-fast eating rate' were significantly associated with a greater odds of CUD. Further prospective investigations are warranted to confirm this association.

\section{Key words: Dietary habits: Chronic uninvestigated dyspepsia: Functional gastrointestinal disorders: Meal regularity:} Eating rates: Fluid intake

Functional dyspepsia (FD) has been defined as 'the presence of symptoms that originate in the gastro-duodenal region, in the absence of any organic, systemic or metabolic disease explaining the symptoms ${ }^{\text {(1) }}$. Symptoms include postprandial fullness, early satiation, or epigastric pain or burning ${ }^{(1)}$. FD is the most common cause of dyspepsia ${ }^{(2)}$. It is an important public health problem, and its prevalence has been estimated to be $20-30$ and $8-23 \%$ in Western and Asian populations, respectively ${ }^{(1,3,4)}$.
A recent systematic review in $\operatorname{Iran}^{(5)}$ has reported that the prevalence of dyspepsia ranges from 2.9 to $29.9 \%$ in different parts of the country. Although not life-threatening, FD reduces quality of life, imposes a considerable burden on the health care system and greatly increases health costs ${ }^{(6-8)}$.

Several contributing factors including age, sex, ethnicity, smoking status, regular use of non-steroidal anti-inflammatory drugs and lifestyle have been shown to be related to dyspepsia ${ }^{(9)}$.

Abbreviations: CUD, chronic uninvestigated dyspepsia; FD, functional dyspepsia; IUMS, Isfahan University of Medical Sciences; LCA, latent class analysis.

*Corresponding author: Dr A. Esmaillzadeh, fax +98 3116682509 , email esmaillzadeh@hlth.mui.ac.ir

†Joint senior author. 
Among lifestyle-related factors, diet may play a central role. Patients with FD frequently report that their symptoms are related to, or exacerbated by, eating (particularly fatty foods) ${ }^{(10-13)}$. Only a few studies ${ }^{(13-16)}$ have evaluated the role of dietary macronutrients. For example, consumption of a high-fat meal has been shown to induce nausea and pain ${ }^{(13)}$, and the effects have been found to be greater when compared with the consumption of a high-carbohydrate meal in FD patients ${ }^{(14)}$. Dietary fibre has also been found to vary in patients with FD, with intake being higher $^{(17)}$, lower ${ }^{(18)}$ or comparable ${ }^{(19)}$ with that of healthy controls.

The available studies have mostly evaluated food intolerances in $\mathrm{FD}^{(17,19,20)}$, and there is little and inconsistent information about dietary behaviour in patients with $\mathrm{FD}^{(17,21-24)}$ For example, while speed of eating, irregular meal patterns, number and size of meals, eating before bedtime and intermeal interval have been associated with an increased risk of FD in some observational studies ${ }^{(21-23)}$, such findings have not been confirmed by others ${ }^{(17,24)}$. While previous studies have assessed certain aspects of dietary behaviours, application of latent class analysis (LCA), a person-centred approach, has not been undertaken. LCA provides a unique opportunity to classify individuals according to behavioural subclasses. The application of this method results in the identification of subjects within each latent class who are highly similar to each other and uniquely different from those in other classes. Therefore, LCA allows for the identification of discrete dietary behaviour groups instead of considering them as a single behaviour in diet-disease relationships. Furthermore, previous studies have mostly been performed in Western countries, and limited data are available in developing countries, where dietary intakes and dietary behaviours may be substantially different from those in developed nations. Given the limited information available, the best dietary management of dyspepsia remains uncertain, and available dietary strategies are limited. Therefore, identification of particular dietary behaviours that are associated with dyspepsia may allow the development of novel dietary treatment approaches for this condition. The aim of the present study was to determine the association between patterns of dietary behaviours, identified by LCA, and chronic uninvestigated dyspepsia (CUD) in a large cohort of Iranian adults.

\section{Materials and methods}

\section{Participants}

The present study was performed within the framework of the Study on the Epidemiology of Psychological, Alimentary Health and Nutrition (SEPAHAN) project, which is a crosssectional study that investigates the epidemiological aspects of functional gastrointestinal disorders and their relationships with lifestyle measures and psychological factors. The study design, sample selection, characteristics of study participants as well as details on data collection methods have been described in Adibi et al. ${ }^{(25)}$. Briefly, the project was carried out by non-academic staff members of the Isfahan University of Medical Sciences (IUMS), Isfahan, Iran, who worked in hospitals, university campus and health centres affiliated with the IUMS. To increase the accuracy of the collected data as well as the response rate, data collection was performed in two phases. In the first phase, a self-administered questionnaire on demographic and lifestyle factors, including nutritional habits and dietary intakes, was used (response rate $86 \cdot 2 \%)$. In the second phase, a self-administered questionnaire containing questions on gastrointestinal function and psychological factors was used (response rate 64.6\%). In the present analysis, we used the data from 4763 adults who had completed the questionnaire on both dietary habits and gastrointestinal symptoms. The Regional Bioethics Committee of IUMS approved the study protocol, and the participants provided written informed consent

\section{Assessment of dietary behaviours}

Based on earlier studies ${ }^{(17,22,23)}$, dietary behaviours were predefined and assessed in four domains ('meal patterns', 'eating rate', 'intra-meal fluid intake' and 'meal-to-sleep interval') using a detailed questionnaire, as summarised in Table 1.

\section{Assessment of chronic uninvestigated dyspepsia}

A modified Persian version of the Rome III questionnaire, as part of the main comprehensive questionnaire, was used for the assessment of functional gastrointestinal disorders, including CUD $^{(25)}$. During the face validation of the questionnaire, we found that most participants were unable to distinguish between the descriptors used in the original Rome III questionnaire (never, less than $1 \mathrm{~d}$ per month, $1 \mathrm{~d}$ per month, 2-3d per month, $1 \mathrm{~d}$ per week, more than $1 \mathrm{~d}$ per week, or every day). Therefore, we modified the rating scales to consist of only four descriptors (i.e. never or rarely, occasionally, often and always) ${ }^{(25)}$. In the present study, individuals with one or more of the following characteristics were defined as having CUD: bothersome postprandial fullness (defined as feeling uncomfortably full after eating a regular-sized meal, often or always); early satiation (defined as being unable to finish a regular-sized meal, often or always); epigastric pain or burning (defined as feeling pain or burning in the middle of the abdomen, often or always). The validity and reliability of the Persian version of the Rome III questionnaire in an Iranian population has been demonstrated previously ${ }^{(26)}$.

\section{Statistical analyses}

The classes (subcategories of each domain) of dietary behaviours were identified by LCA, as detailed in Table 1. LCA examines the pattern of relationships among a set of observed categorical or continuous variables, and classifies individuals with similar behaviours into latent classes ${ }^{(27)}$. We used four main domains of dietary behaviours, each containing different indicators, as shown in Table 1. For each domain of dietary behaviour, we first applied a one-class model. Then, we sequentially increased the number of latent classes in order to determine the most parsimonious model. The interpretability of the obtained classes was also taken into account. For example, in the case of meal patterns, if a high percentage 
Table 1. Questions used in latent class analysis for the participants of the SEPAHAN (Study on the Epidemiology of Psychological, Alimentary Health and Nutrition)

\begin{tabular}{|c|c|c|c|c|}
\hline \multicolumn{5}{|l|}{ Meal pattern } \\
\hline How many meals do you eat per $d ?$ & 1 & \multicolumn{2}{|c|}{2} & 3 \\
\hline How often do you eat your meals regularly? & Never & Occasionally & Often & Always \\
\hline How often do you eat breakfast during a week? & Never or once/week & 2-4 times/week & 5-6 times/week & Every day \\
\hline How often do you eat lunch during a week? & Never or once/week & 2-4 times/week & $5-6$ times/week & Every day \\
\hline How often do you eat dinner during a week? & Never or once/week & 2-4 times/week & $5-6$ times/week & Every day \\
\hline \multicolumn{5}{|l|}{ Eating rate } \\
\hline How thoroughly do you chew food? & Not very well & \multicolumn{2}{|c|}{ Well } & \multirow{3}{*}{$\begin{array}{l}\text { Very well } \\
>20 \text { min } \\
>20 \text { min }\end{array}$} \\
\hline How long does it take you to eat lunch? & Never eat lunch & $<10 \min$ & $10-20 \mathrm{~min}$ & \\
\hline How long does it take you to eat dinner? & Never eat dinner & $<10 \min$ & $10-20 \mathrm{~min}$ & \\
\hline \multicolumn{5}{|l|}{ Intra-meal fluid intake } \\
\hline $\begin{array}{l}\text { What is the temperature of fluids you drink with } \\
\text { meals? }\end{array}$ & Not too cold & \multicolumn{2}{|c|}{ Cold } & Very cold \\
\hline $\begin{array}{l}\text { How often do you drink fluids with meals or } \\
\text { immediately before and after meals? }\end{array}$ & Never & Occasionally & Often & Always \\
\hline $\begin{array}{l}\text { How many glasses of fluids do you drink } \\
\text { during meals? }\end{array}$ & $\leq 1$ glass & $2-3$ glasses & $3-4$ glasses & $\geq 4$ glasses \\
\hline \multicolumn{5}{|l|}{ Meal-to-sleep interval } \\
\hline $\begin{array}{l}\text { How long does it take you to go to bed (or to lie } \\
\text { down) after lunch? }\end{array}$ & Never sleep & $<30 \min$ & $0.5-<2 h$ & $2-4 \mathrm{~h}$ \\
\hline $\begin{array}{l}\text { How long does it take you to go to bed (or to lie } \\
\text { down) after dinner? }\end{array}$ & $<30 \min$ & $0.5-<2 h$ & $2-4 h$ & $>4 \mathrm{~h}$ \\
\hline $\begin{array}{l}\text { How long does it take you to go to bed (or to lie } \\
\text { down) following the last snack after dinner? }\end{array}$ & Never eat snack & $<30 \min$ & $0 \cdot 5-2 h$ & $>2 \mathrm{~h}$ \\
\hline
\end{tabular}

of subjects responded that they eat three meals per $d$, eat meals regularly and eat breakfast, and eat lunch and dinner every day, such a pattern of dietary behaviour was considered as interpretable and was defined as a 'regular meal pattern'. During the application of LCA, those questions that did not significantly contribute to the construction of exclusive classes were excluded. Finally, based on the constructed classes for each dietary behaviour, the class memberships of the subjects were determined (i.e. four new binary or multinomial variables). These variables were used as the predictors of CUD for further analyses.

One-way ANOVA was used to compare continuous variables between the classes of dietary behaviours. Distribution of individuals in terms of categorical variables across the classes of dietary behaviours was examined using the $\chi^{2}$ test. To determine the association between patterns of dietary behaviours and CUD, we used logistic regression analysis in different models. In other words, to find an independent association between each domain of dietary behaviours and CUD, we controlled for the mutual effects of the other dietary behaviours in the first model. Then, we added age and sex into the analysis. Further adjustments were made for physical activity and smoking status in the third model. We additionally controlled for BMI in the next model. Finally, we removed the other dietary behaviours from the model to determine the combined effects of dietary behaviours on CUD. In addition to the analyses for the whole population, we also conducted stratified analyses by sex and BMI status $\left(<25\right.$ and $\left.\geq 25 \mathrm{~kg} / \mathrm{m}^{2}\right)$.

LCA was performed using $\mathrm{R}$ free statistical software version 2.15.1 (R Foundation for Statistical Computing). All the other analyses were performed using the Statistical Package for Social Sciences (version 16.0; SPSS, Inc.). $P$ values $<0.05$ (two-sided) were considered statistically significant.

\section{Results}

CUD was identified in $15 \cdot 2 \%$ (95\% CI $14 \cdot 1,16 \cdot 2 \% ; n$ 723) of patients. The occurrence of early satiation, bothersome postprandial fullness, and epigastric pain or burning was $6.3 \%$ ( $n$ 302), 8.0\% ( $n$ 384) and 7.8\% ( $n$ 371), respectively. The latent classes identified by LCA, the distribution of participants (class size) and their responses to each question (item-specific response patterns in each class) in the four domains are presented in Table 2. For meal patterns across the entire population, two main classes were identified: 'regular' ( $n$ 2953, 62\%) and 'irregular' ( $n$ 1810, 38\%). For eating rate, three distinct classes were obtained: 'moderate' ( $n$ 3144, 66\%); 'moderate-to-slow' ( $n$ 1143, 24\%); 'moderate-to-fast' ( $n$ 476, 10\%). In terms of intra-meal fluid intake, participants were categorised into two major classes: 'moderate intra-meal drinking' ( $n$ 2620, $55 \%$ ) and 'high intra-meal drinking' ( $n$ 2143, 45\%). In terms of the interval between meals and sleeping, two distinct classes were obtained: 'short meal-to-sleep interval' ( $n$ 3477, $73 \%$ ) and 'long meal-to-sleep interval' ( $n$ 1286, $27 \%$ ).

The general characteristics of the study participants across the different classes of dietary behaviours are provided in Table 3. Compared with those with a 'moderate eating rate', subjects with a 'moderate-to-fast eating rate' had higher weight and BMI. Those with a 'moderate-to-slow eating rate' tended to be older and more physically active compared with others. Subjects who drank more fluids with meals had higher BMI and weight and were less likely to be older, women and university-educated compared with those who were 'moderate fluid drinkers'. Participants with an 'irregular meal pattern' were more likely to be women, had higher BMI, prevalent CUD and epigastric pain compared with those who had a 'regular meal pattern'. Subjects with a 'long meal-to-sleep interval' were more likely to be single 
Table 2. Latent classes identified by latent class analysis for the participants of the SEPAHAN (Study on the Epidemiology of Psychological, Alimentary Health and Nutrition)

Type of dietary behaviours and their item-specific response patterns

Class-specific response patterns of participants

Meal pattern...

\begin{tabular}{lc}
\hline Regular & Irregular \\
\hline 0.62 & 0.38 \\
0 & 0.09 \\
0.0067 & 0.66 \\
0.9933 & 0.25 \\
& \\
0.05 & 0.14 \\
0.27 & 0.40 \\
0.47 & 0.37 \\
0.21 & 0.09 \\
& \\
0.01 & 0.18 \\
0.06 & 0.27 \\
0.09 & 0.13 \\
0.83 & 0.40 \\
& \\
0 & 0.02 \\
0.0004 & 0.07 \\
0.01 & 0.12 \\
0.99 & 0.78 \\
0.0068 & 0.19 \\
0.04 & 0.28 \\
0.12 & 0.20 \\
0.83 & 0.32 \\
\hline
\end{tabular}

Class size

How many meals do you eat per d?

1

3

How often do you eat your meals regularly?

Never

Occasionally

Often

Always

How often do you eat breakfast during a week?

Never or once/week

2-4 times/week

5-6 times/week

Every day

How often do you eat lunch during a week?

Never or once/week

2-4 times/week

5-6 times/week

Every day

How often do you eat dinner during a week?

Never or once/week

2-4 times/week

0.32

Every day

\begin{tabular}{|c|c|c|c|}
\hline Eating rate... & Moderate & Moderate-to-slow & Moderate-to-fast \\
\hline Class size & 0.66 & 0.24 & $0 \cdot 10$ \\
\hline \multicolumn{4}{|c|}{ How thoroughly do you chew food? } \\
\hline Not very well & 0.13 & 0.04 & 0.46 \\
\hline Well & 0.76 & 0.69 & 0.53 \\
\hline Very well & $0 \cdot 1$ & 0.27 & 0.01 \\
\hline \multicolumn{4}{|c|}{ How long does it take you to eat lunch? } \\
\hline Never eat & 0 & 0.03 & 0 \\
\hline$<10 \min$ & 0.07 & 0 & 0.99 \\
\hline $10-20 \mathrm{~min}$ & 0.92 & $0 \cdot 21$ & 0.01 \\
\hline$>20 \min$ & 0.0021 & 0.76 & 0 \\
\hline \multicolumn{4}{|c|}{ How long does it take you to eat dinner? } \\
\hline Never eat dinner & 0.04 & 0.23 & 0 \\
\hline$<10 \min$ & 0.18 & 0.06 & 0.96 \\
\hline $10-20 \mathrm{~min}$ & 0.66 & 0.50 & 0.03 \\
\hline$>20 \mathrm{~min}$ & 0.1 & 0.2 & 0.0001 \\
\hline
\end{tabular}

Intra-meal fluid intake. .

Moderate intra-meal drinking

High intra-meal drinking

\begin{tabular}{|c|c|c|}
\hline Class size & 0.55 & 0.45 \\
\hline \multicolumn{3}{|l|}{$\begin{array}{l}\text { How often do you drink fluids with meals or immediately } \\
\text { before and after meals? }\end{array}$} \\
\hline Never & 0.11 & 0.0043 \\
\hline Occasionally & 0.60 & 0.15 \\
\hline Often & 0.24 & 0.38 \\
\hline Always & 0.04 & 0.46 \\
\hline \multicolumn{3}{|l|}{ How many glasses of fluids do you drink during meals? } \\
\hline$\leq 1$ glass & 0.92 & 0.39 \\
\hline $2-3$ glasses & 0.08 & 0.50 \\
\hline $3-4$ glasses & 0.0008 & 0.07 \\
\hline$\geq 4$ glasses & 0 & 0.02 \\
\hline \multicolumn{3}{|l|}{ What is the temperature of fluids you drink with meals? } \\
\hline Not too cold & 0.65 & $0 \cdot 36$ \\
\hline Cold & 0.34 & 0.60 \\
\hline Very cold & 0.0059 & 0.03 \\
\hline
\end{tabular}

Short meal-to-sleep interval Long meal-to-sleep interval

Meal-to-sleep interval. . .

0.73

$0 \cdot 27$

Class size

How long does it take you to go to bed (or to lie down) after lunch?

Never sleep

$<30 \mathrm{~min}$ 
Table 2. Continued

Type of dietary behaviours and their item-specific response patterns

Meal pattern. .

$0.5-<2 h$

$2-4 \mathrm{~h}$

$>4 \mathrm{~h}$

How long does it take you to go to bed (or to lie down)

after dinner?

$<30 \mathrm{~min}$

$0.5-<2 h$

$2-4 \mathrm{~h}$

$>4 \mathrm{~h}$

How long does it take you to go to bed (or to lie down)

following the last snack after dinner?

Never eat snacks

$<30 \mathrm{~min}$

$0.5-2 h$

$>2 \mathrm{~h}$
Class-specific response patterns of participants

\begin{tabular}{lc} 
Regular & Irregular \\
\hline 0.30 & 0.38 \\
0.05 & 0.09 \\
0.0093 & 0.02
\end{tabular}

$0.03 \quad 0.0011$

$0.49 \quad 0.10$

$0.44 \quad 0.57$

$0.04 \quad 0.32$

$0.28 \quad 0$

$0.27 \quad 0$

$0.45 \quad 0.59$

$0.0009 \quad 0.4$

and physically active compared with those with a 'short mealto-sleep interval'.

Multivariable-adjusted OR for CUD across the different classes of dietary behaviours are presented in Table 4. After adjustment for age, sex and the mutual effects of the other dietary behaviours, the 'irregular meal pattern' was significantly associated with a greater odds of CUD (OR 1.41, 95\% CI $1.15,1.73)$. This association remained significant even after further controlling for smoking status and physical activity (OR 1.38, 95\% CI 1.10, 1.73). When BMI was taken into account, the association did not change (OR 1.42, 95\% CI 1.12, 1.78). After removing the other dietary behaviours from the model, we found that individuals with an 'irregular meal pattern' were $42 \%$ (OR 1.42, $95 \%$ CI 1.15, 1.75) more likely to have CUD compared with those who had a 'regular meal pattern'. We found that the 'moderate-to-fast eating rate' was not associated with the risk of CUD. However, when BMI was taken into account, the association became just significant (OR 1.41, 95\% CI 1.00, 1.99). Exclusion of the other dietary behaviours slightly strengthened this association (OR 1.49, 95\% CI 1.09, 2.03). No significant associations were found between the patterns of 'meal-to-sleep interval' and 'intra-meal fluid intake' and CUD.

When we examined the associations separately for men and women (Table 5), we found that men with a 'moderate-to-fast eating rate' were more likely to have CUD compared with those who had a 'moderate eating rate' (OR 2.22, 95\% CI $1.31,3.76)$. No other significant associations were found between dietary behaviours and CUD in men. The 'irregular meal pattern' was associated with a greater odds of CUD in women even after taking into account potential confounders such as age, sex, physical activity, smoking status, BMI and the mutual effects of the other dietary behaviours (OR 1.48, $95 \%$ CI $1 \cdot 12,1.95)$.

Stratified analyses by BMI status revealed that normalweight individuals with an 'irregular meal pattern' tended to have a greater odds of CUD compared with their counterparts who had a 'regular meal pattern' (OR 1·55, $95 \%$ CI 1·15, 2·10; Table 6). 'High intra-meal fluid drinking' was marginally associated with a higher odds of CUD compared with 'moderate drinking' in normal-weight subjects (OR 1.33, $95 \%$ CI 0.99, 1.78). We also found that overweight individuals with a 'moderate-to-fast eating rate' were more likely to have CUD compared with those who had a 'moderate eating rate' (OR 1.74, 95\% CI 1.07, 2.83). No further associations were found between dietary behaviours and CUD either in normal- or overweight participants.

\section{Discussion}

The present study provides novel insights into the association of dietary factors in CUD. We found a significant positive association between the 'irregular meal pattern' and the risk of CUD. This was independent of potential confounders and the effect of other dietary behaviours. The 'moderate-to-fast eating rate' was significantly associated with an increased risk of CUD after taking potential confounders into account. In contrast, the 'long meal-to-sleep interval' was not related to CUD, and no significant relationship was observed between 'intra-meal fluid intake' and the risk of CUD. To our knowledge, this is the first epidemiological study in which distinct classes of dietary behaviours identified by LCA have been related to CUD. The Rome III diagnostic criterion for FD requires endoscopic examination to exclude organic diseases. As we could not perform endoscopic evaluations, we assessed the association between dietary behaviours and CUD. However, it should be noted that here we compare our findings with other investigations that evaluate FD.

Dyspepsia affects a huge number of people worldwide. Although not life-threatening, this condition reduces quality of life to an extent comparable with mild heart failure and menopause ${ }^{(8)}$. Due to the heterogeneous nature of functional gastrointestinal disorders, their underlying causes have not been thoroughly identified, and no effective medication is available for their treatment. Genetic factors are involved in the aetiology of functional gastrointestinal disorders ${ }^{(28)}$; however, lifestyle-related factors are assumed to play a key modulator role ${ }^{(29)}$. Although a large proportion of patients with FD report that their symptoms are related to, or exacerbated after, meal intake ${ }^{(23,30)}$, the role of both dietary intake and dietary 
Table 3. General characteristics of the study participants across the different classes of dietary behaviours in the SEPAHAN (Study on the Epidemiology of Psychological, Alimentary Health and Nutrition)

(Mean values and standard deviations)

\begin{tabular}{|c|c|c|c|c|c|c|c|c|c|c|c|c|c|c|c|c|c|c|c|c|c|c|}
\hline & \multicolumn{4}{|c|}{ Meal pattern } & \multirow[b]{3}{*}{$P^{*}$} & \multicolumn{6}{|c|}{ Eating rate } & \multicolumn{5}{|c|}{ Intra-meal fluid intake } & \multicolumn{5}{|c|}{ Meal-to-sleep-interval } & \multirow[b]{3}{*}{$P^{*}$} \\
\hline & \multicolumn{2}{|c|}{ Regular } & \multicolumn{2}{|c|}{ Irregular } & & \multicolumn{2}{|c|}{ Moderate } & \multicolumn{2}{|c|}{$\begin{array}{l}\text { Moderate- } \\
\text { to-slow }\end{array}$} & \multicolumn{2}{|c|}{$\begin{array}{l}\text { Moderate- } \\
\text { to-fast }\end{array}$} & \multirow[b]{2}{*}{$P^{*}$} & \multicolumn{2}{|c|}{$\begin{array}{l}\text { Moderate } \\
\text { intra-meal } \\
\text { drinking }\end{array}$} & \multicolumn{2}{|c|}{$\begin{array}{c}\text { High } \\
\text { intra-meal } \\
\text { drinking }\end{array}$} & \multirow[b]{2}{*}{$P^{*}$} & \multicolumn{2}{|c|}{$\begin{array}{l}\text { Short } \\
\text { meal-to- } \\
\text { sleep }\end{array}$} & \multicolumn{2}{|c|}{$\begin{array}{l}\text { Long } \\
\text { meal-to- } \\
\text { sleep }\end{array}$} & \\
\hline & Mean & SD & Mean & SD & & Mean & SD & Mean & SD & Mean & SD & & Mean & SD & Mean & SD & & Mean & SD & Mean & SD & \\
\hline Age (years) & $36 \cdot 3$ & 8.1 & $36 \cdot 8$ & $8 \cdot 1$ & 0.07 & $36 \cdot 1$ & 7.9 & 37.9 & 8.6 & $36 \cdot 4$ & $7 \cdot 8$ & 0.001 & 36.9 & $8 \cdot 1$ & 35.7 & $8 \cdot 1$ & $<0.001$ & 36.5 & 8 & $36 \cdot 6$ & 8.4 & 0.92 \\
\hline Weight (kg) & 68.6 & 12.5 & 68.6 & $12 \cdot 8$ & 0.91 & $68 \cdot 3$ & 12.5 & 68.9 & 13 & 71.4 & 13 & 0.001 & 67.6 & $12 \cdot 3$ & 70.5 & 13 & $<0.001$ & 68.5 & 12.5 & 68.8 & 13 & 0.61 \\
\hline $\mathrm{BMI}\left(\mathrm{kg} / \mathrm{m}^{2}\right)$ & 24.7 & 3.7 & 25.07 & 4 & 0.01 & $24 \cdot 8$ & 3.8 & $24 \cdot 8$ & 3.9 & $25 \cdot 6$ & $4 \cdot 1$ & 0.001 & 24.8 & 3.8 & 25.09 & 3.9 & 0.02 & 24.9 & 3.8 & 24.9 & 3.9 & 0.91 \\
\hline Female (\%) & \multicolumn{2}{|c|}{53} & \multicolumn{2}{|c|}{64} & $<0.001$ & \multicolumn{2}{|c|}{59} & \multicolumn{2}{|c|}{51} & \multicolumn{2}{|c|}{51} & 0.001 & \multicolumn{2}{|c|}{62} & \multicolumn{2}{|c|}{50} & $<0.001$ & \multicolumn{2}{|c|}{57} & \multicolumn{2}{|c|}{57} & 0.87 \\
\hline Married $(\%)$ & \multirow{2}{*}{\multicolumn{2}{|c|}{$\begin{array}{c}82 \\
3\end{array}$}} & \multirow{2}{*}{\multicolumn{2}{|c|}{$\begin{array}{c}80 \\
4\end{array}$}} & 0.04 & \multirow{2}{*}{\multicolumn{2}{|c|}{$\begin{array}{c}81 \\
3\end{array}$}} & \multirow{2}{*}{\multicolumn{2}{|c|}{81}} & 82 & & 0.92 & 81 & & 8 & & 0.85 & 8 & & 7) & & 0.001 \\
\hline Current smokers (\%) & & & & & $0 \cdot 17$ & & & & & 3 & & 0.06 & 3 & & 4 & & 0.59 & 3 & & 4 & & 0.72 \\
\hline $\begin{array}{l}\text { Physical activity } \\
\geq 1 \mathrm{~h} / \mathrm{d}(\%)\end{array}$ & 35 & & 3 & & 0.66 & 33 & & 40 & & 32 & & 0.001 & 34 & & 3 & & 0.41 & 3 & & 4 & & 0.001 \\
\hline $\begin{array}{l}\text { University education (\%) } \\
\text { Gastrointestinal } \\
\text { disorders (\%) }\end{array}$ & $5 \varsigma$ & & 5 & & $0 \cdot 19$ & 61 & & 50 & & 55 & & 0.001 & 61 & & 5 & & $<0.001$ & 58 & & $5 \varepsilon$ & & $0 \cdot 76$ \\
\hline $\begin{array}{l}\text { Chronic uninvestigated } \\
\text { dyspepsiat }\end{array}$ & 13 & & 1 & & $<0.001$ & 15 & & 14 & & 18 & & 0.13 & 14 & & 1 & & 0.11 & 1 & & 1 & & 0.64 \\
\hline Postprandial fullness $\ddagger$ & 7 & & 1 & & $<0.001$ & 8 & & 8 & & 9 & & 0.74 & 8 & & 9 & & 0.17 & 8 & & 7 & & 0.65 \\
\hline $\begin{array}{l}\text { Epigastric pain or } \\
\text { burning§ }\end{array}$ & 7 & & 9 & & 0.004 & 8 & & 8 & & 9 & & 0.38 & 8 & & 8 & & 0.67 & 8 & & 6 & & 0.05 \\
\hline Early satiation\| & 55 & & 4 & & $<0.001$ & 69 & & 19 & & 12 & & 0.75 & 59 & & 6 & & 0.30 & 8 & & 17 & & 0.58 \\
\hline
\end{tabular}

${ }^{*} P$ values were determined using ANOVA for continuous variables and $\chi^{2}$ test for categorical variables.

†Participants with chronic uninvestigated dyspepsia were defined according to the Rome III diagnostic criteria as having one or more of the following characteristics: bothersome postprandial fullness (often or always); early

satiation (often or always); epigastric pain or burning (often or always).
† Postprandial fullness was defined as feeling uncomfortably full after eating a regular-sized meal, often or always.

$\S$ Epigastric pain or burning was defined as feeling pain or burning in the middle of the abdomen, often or always.

Early satiation was defined as being unable to finish a regular-sized meal, often or always. 
Table 4. Multivariable-adjusted OR for chronic uninvestigated dyspepsia across the different classes of dietary behaviours for the participants of the SEPAHAN (Study on the Epidemiology of Psychological, Alimentary Health and Nutrition)

(Odds ratios and $95 \%$ confidence intervals)

\begin{tabular}{|c|c|c|c|c|c|c|c|c|c|c|c|c|c|c|}
\hline & \multicolumn{3}{|c|}{ Meal pattern } & \multicolumn{5}{|c|}{ Eating rate } & \multicolumn{3}{|c|}{ Intra-meal fluid intake } & \multicolumn{3}{|c|}{ Meal-to-sleep-interval } \\
\hline & \multirow[b]{2}{*}{ Regular } & \multicolumn{2}{|c|}{ Irregular } & \multirow[b]{2}{*}{ Moderate } & \multicolumn{2}{|c|}{ Moderate-to-slow } & \multicolumn{2}{|c|}{ Moderate-to-fast } & \multirow{2}{*}{$\begin{array}{l}\text { Moderate intra- } \\
\text { meal drinking }\end{array}$} & \multicolumn{2}{|c|}{$\begin{array}{l}\text { High intra-meal } \\
\text { drinking }\end{array}$} & \multirow{2}{*}{$\begin{array}{c}\text { Short meal- } \\
\text { to-sleep }\end{array}$} & \multicolumn{2}{|c|}{$\begin{array}{l}\text { Long meal- } \\
\text { to-sleep }\end{array}$} \\
\hline & & OR & $95 \% \mathrm{Cl}$ & & OR & $95 \% \mathrm{Cl}$ & OR & $95 \% \mathrm{Cl}$ & & OR & $95 \% \mathrm{Cl}$ & & OR & $95 \% \mathrm{Cl}$ \\
\hline \multicolumn{15}{|c|}{ Chronic uninvestigated dyspepsia* } \\
\hline Crude model & 1.00 & 1.53 & $1.29,1.81$ & 1.00 & 0.95 & $0.77,1.17$ & 1.26 & $0.98,1.63$ & 1.00 & $1 \cdot 14$ & $0.96,1.35$ & 1.00 & 0.94 & $0.75,1 \cdot 18$ \\
\hline Model $1 \dagger$ & 1.00 & 1.51 & $1.25,1.82$ & 1.00 & 0.94 & $0.74,1.19$ & 1.22 & $0.92,1.61$ & 1.00 & 1.19 & $0.99,1.43$ & 1.00 & 0.93 & $0.72,1.18$ \\
\hline Model 2‡ & 1.00 & 1.41 & $1.15,1.73$ & 1.00 & 1.02 & $0.79,1.32$ & 1.29 & $0.95,1.76$ & 1.00 & 1.18 & $0.96,1.45$ & 1.00 & 0.94 & $0.72,1.22$ \\
\hline Model $3 \S$ & 1.00 & 1.38 & $1 \cdot 10,1.73$ & 1.00 & 0.99 & $0.74,1.32$ & 1.32 & $0.94,1.85$ & 1.00 & $1 \cdot 20$ & $0.96,1.5$ & 1.00 & 0.92 & $0.69,1.24$ \\
\hline Model $4 \|$ & 1.00 & 1.42 & $1.12,1.78$ & 1.00 & 0.96 & $0.71,1.30$ & 1.41 & $1.00,1.99$ & 1.00 & 1.21 & $0.96,1.52$ & 1.00 & 0.93 & $0.69,1.26$ \\
\hline Model 59 & 1.00 & 1.42 & $1 \cdot 15,1.75$ & 1.00 & 0.98 & $0.75,1.28$ & 1.49 & $1.09,2.03$ & 1.00 & 1.17 & $0.95,1.45$ & 1.00 & 1.01 & $0.76,1.33$ \\
\hline
\end{tabular}

"Patients with chronic uninvestigated dyspepsia were defined according to the Rome III diagnostic criteria as having one or more of the following characteristics: bothersome postprandial fullness (often or always); early satiation (often or always); epigastric pain or burning (often or always).

† Model 1 was adjusted for other dietary behaviours.

† Model 2 was adjusted for other dietary behaviours, age and sex.

Model 3 was adjusted for other dietary behaviours, age, sex, physical activity and smoking status.

|| Model 4 was adjusted for other dietary behaviours, age, sex, physical activity, smoking status and BM

I Model 5 was adjusted for age, sex, physical activity, smoking status and BMI.

Table 5. Multivariable-adjusted OR for chronic uninvestigated dyspepsia across the different classes of dietary behaviours stratified by sex for the participants of the SEPAHAN (Study on the Epidemiology of Psychological, Alimentary Health and Nutrition)

(Odds ratios and $95 \%$ confidence intervals)

\begin{tabular}{|c|c|c|c|c|c|c|c|c|c|c|c|c|c|c|}
\hline & \multicolumn{3}{|c|}{ Meal pattern } & \multicolumn{5}{|c|}{ Eating rate } & \multicolumn{3}{|c|}{ Intra-meal fluid intake } & \multicolumn{3}{|c|}{ Meal-to-sleep interval } \\
\hline & \multirow[b]{2}{*}{ Regular } & \multicolumn{2}{|c|}{ Irregular } & \multirow[b]{2}{*}{ Moderate } & \multicolumn{2}{|c|}{ Moderate-to-slow } & \multicolumn{2}{|c|}{ Moderate-to-fast } & \multirow{2}{*}{$\begin{array}{l}\text { Moderate intra- } \\
\text { meal drinking }\end{array}$} & \multicolumn{2}{|c|}{$\begin{array}{l}\text { High intra-meal } \\
\text { drinking }\end{array}$} & \multirow{2}{*}{$\begin{array}{c}\text { Short meal- } \\
\text { to-sleep }\end{array}$} & \multicolumn{2}{|c|}{$\begin{array}{l}\text { Long meal- } \\
\text { to-sleep }\end{array}$} \\
\hline & & OR & $95 \% \mathrm{Cl}$ & & OR & $95 \% \mathrm{Cl}$ & OR & $95 \% \mathrm{Cl}$ & & OR & $95 \% \mathrm{Cl}$ & & OR & $95 \% \mathrm{Cl}$ \\
\hline \multicolumn{15}{|l|}{ Men } \\
\hline Crude model & 1.00 & 1.45 & $1.08,1.96$ & 1.00 & 1.17 & $0.84,1.64$ & 1.78 & $1 \cdot 21,2 \cdot 61$ & 1.00 & 1.20 & $0.90,1.6$ & 1.00 & 1.03 & $0.70,1.5$ \\
\hline Model $1^{\star}$ & 1.00 & 1.50 & $1.08,2.08$ & 1.00 & 1.24 & $0.84,1.82$ & 1.73 & $1 \cdot 13,2 \cdot 65$ & 1.00 & 1.18 & $0.86,1.61$ & 1.00 & 1.00 & $0.66,1.51$ \\
\hline Model $2 \dagger$ & 1.00 & 1.36 & $0.94,1.97$ & 1.00 & 1.30 & $0.85,1.97$ & 1.69 & $1.04,2.75$ & 1.00 & 1.18 & $0.83,1.67$ & 1.00 & 0.88 & $0.55,1.42$ \\
\hline Model $3 \ddagger$ & 1.00 & 1.18 & $0.79,1.75$ & 1.00 & 1.42 & $0.91,2 \cdot 22$ & 1.88 & $1.13,3.14$ & 1.00 & 1.24 & $0.85,1.8$ & 1.00 & 0.89 & $0.53,1.48$ \\
\hline Model $4 \S$ & 1.00 & 1.25 & $0.83,1.90$ & 1.00 & 1.40 & $0.87,2.25$ & $2 \cdot 22$ & $1.31,3.76$ & 1.00 & 1.23 & $0.83,1.82$ & 1.00 & 0.90 & $0.52,1.56$ \\
\hline Model $5 \|$ & 1.00 & 1.21 & $0.84,1.75$ & 1.00 & 1.27 & $0.84,1.91$ & $2 \cdot 14$ & $1.34,3.4$ & 1.00 & 1.22 & $0.86,1.73$ & 1.00 & 0.99 & $0.61,1.61$ \\
\hline \multicolumn{15}{|l|}{ Women } \\
\hline Crude model & 1.00 & 1.47 & $1.19,1.82$ & 1.00 & 0.85 & $0.64,1.13$ & 1.14 & $0.80,1.60$ & 1.00 & 1.23 & $0.99,1.53$ & 1.00 & 0.95 & $0 \cdot 71,1.26$ \\
\hline Model 1 & 1.00 & 1.42 & $1.13,1.79$ & 1.00 & 0.82 & $0.59,1.12$ & 1.08 & $0.74,1.58$ & 1.00 & 1.30 & $1.02,1.65$ & 1.00 & 0.92 & $0.68,1.26$ \\
\hline Model 2 & 1.00 & 1.43 & $1.12,1.83$ & 1.00 & 0.90 & $0.64,1.24$ & 1.12 & $0.75,1.66$ & 1.00 & 1.19 & $0.92,1.52$ & 1.00 & 0.97 & $0.7,1.35$ \\
\hline Model 3 & 1.00 & 1.48 & $1.13,1.95$ & 1.00 & 0.79 & $0.54,1.16$ & 1.05 & $0.66,1.65$ & 1.00 & 1.18 & $0.89,1.56$ & 1.00 & 0.95 & $0.66,1.37$ \\
\hline Model 4 & 1.00 & 1.48 & $1.12,1.95$ & 1.00 & 0.78 & $0.53,1 \cdot 16$ & 1.07 & $0.67,1.71$ & 1.00 & $1 \cdot 21$ & $0.91,1.61$ & 1.00 & 0.96 & $0.66,1.38$ \\
\hline Model 5 & 1.00 & 1.52 & $1.18,1.97$ & 1.00 & 0.84 & $0.59,1 \cdot 19$ & 1.17 & $0.77,1.79$ & 1.00 & 1.16 & $0.89,1.51$ & 1.00 & 1.02 & $0.73,1.44$ \\
\hline
\end{tabular}

* Model 1 was adjusted for other dietary behaviours.

† Model 2 was adjusted for other dietary behaviours and age.

¥Model 3 was adjusted for other dietary behaviours, age, physical activity and smoking status.

$\S$ Model 4 was adjusted for other dietary behaviours, age, physical activity, smoking status and BMI.

|l Model 5 was adjusted for age, physical activity, smoking status and BMI. 
behaviours, including meal patterns, eating rate, intra-meal fluid intake and meal-to-sleep interval, have, to date, not received sufficient attention and, thus, require further consideration.

In the present study, an irregular meal pattern was associated with a greater risk of having CUD. This finding is in line with the results of a population-based cross-sectional study in which irregular intake of meals was related to the symptoms of dyspepsia ${ }^{(21)}$. However, that study did not use LCA to identify dietary behaviours and did not take into account the effect of other variables. The association of an irregular meal pattern with irritable bowel syndrome and FD has also been reported in a case-control study in Japan ${ }^{(31)}$ and by others ${ }^{(20,32)}$. Not all studies have detected a significant association between meal patterns and $\mathrm{FD}^{(17,24)}$; this discrepancy may be due to the case-control design and small sample sizes of these studies. Furthermore, such studies have not matched cases and controls for age, marital status and socio-economic status, all of which may confound dietary behaviours ${ }^{(33,34)}$. In addition, the influence of other dietary behaviours including eating rate, intra-meal fluid intake and meal-to-sleep interval has not been controlled in these studies. It has been shown that patients with FD ate fewer full meals compared with healthy individuals, and, instead, consumed a more smaller meal ${ }^{(23)}$. These findings suggest that patients with FD may reduce their meal size and/or eat irregularly in an attempt to alleviate their symptoms.

We also found a significant association between eating rate and the risk of CUD. Similar to the present results, Sinn et al. ${ }^{(22)}$ found that a higher percentage of women with FD had a faster eating rate than healthy control subjects. Theoretically, fast eating may overload normal reflex responses such as fundic relaxation, which, if impaired for other reasons, may lead to dyspepsia. However, not all studies agree. Carvalho et al. ${ }^{(17)}$ showed that the same percentage of patients with FD and healthy subjects regarded themselves as fast eaters. However, interpretation of their results is difficult, because no clear definition of 'fast eater' was provided. In the study of Sinn et al. ${ }^{(22)}$, fast eating obtained from the questionnaires could not be confirmed when the investigators assessed it objectively. It must also be kept in mind that in the present study, participants perceived themselves as fast eaters. To what extent this could reflect truly fast eating needs to be further assessed.

We did not find significant associations between the pattern of intra-meal fluid intake or meal-to-sleep interval and CUD. These observations are in accordance with the limited available data from previous investigations. Earlier studies have found that the duration of the overnight fast and the percentage of individuals who went to bed soon after having their meal did not differ significantly between patients with FD and controls ${ }^{(17)}$. We did not find any evidence supporting a link between intra-meal fluid intake and CUD, in line with the only other investigation quantifying drinking episodes in patients with FD and healthy subjects ${ }^{(23)}$.

The present data also provide evidence that the associations between dietary behaviours and CUD are modified by sex and BMI. Men with a 'moderate-to-fast eating rate' were more likely to have CUD compared with those who had a 
'moderate eating rate'. Notably, an 'irregular meal pattern' was associated with a greater odds of CUD only in women. The influence of sex or BMI on these associations has not been investigated previously.

It is important to recognise some strengths, as well as potential limitations, of the present study. A major strength is the application of LCA to define discrete dietary behaviours instead of considering them as a single behaviour in dietdisease relationships. Furthermore, the associations reported in the present study were probably independent of other dietary behaviour categories due to controlling for the mutual effects in statistical models. The large sample size is the strength of the present study. Limitations include the crosssectional nature of the study, as a result of which we cannot infer cause-effect relationships from our findings. Furthermore, there is no clear-cut evidence that symptoms influence dietary habits. This may be the case in some patients, but not in others ${ }^{(23,24)}$. To identify the real association between symptoms of dyspepsia and dietary behaviours, further prospective cohort investigations are required. However, due to budget restriction, we did not assess such relationship within the framework of a large prospective cohort. Participants of the present study were 20 to 50 years of age and non-academic staff members of the IUMS. Since the participants in the present study were working in health centres, they may have a healthier lifestyle. Thus, extrapolation of the present findings to the general population in Iran, as well as to other cultural backgrounds, must be done with caution. Despite the adjustment for several lifestyle variables, residual confounding cannot be excluded. Many lifestyle factors such as coffee consumption and emotional experience, which were not controlled for in the present study, may also contribute to the occurrence of dyspepsia. It should also be kept in mind that all information that we used in the present analysis was collected by self-administered questionnaires, and this method of data collection might misclassify participants. However, this is a problem in all epidemiological studies. These misclassifications would move the OR to non-significant associations. Considering the associations that we found, it seems that these associations are much stronger in reality.

In conclusion, we found that dietary behaviours identified by LCA, in particular 'irregular meal pattern' and 'fast eating rate', are associated with an increased risk of CUD. These associations were modified by sex and BMI, such that men with a 'moderate-to-fast eating rate' were more likely to have CUD compared with those who had a 'moderate eating rate', while an 'irregular meal pattern' was associated with a greater odds of CUD only in women. Further prospective investigations are required to explore these findings and the links to the pathophysiology.

\section{Acknowledgements}

The present study was supported by a grant from the Isfahan University of Medical Sciences, Isfahan, Iran

The authors' contributions are as follows: A. H. K., A. E. and P. A. contributed to the conception and design of the study, data collection, statistical analysis and drafting of the manuscript; A. F. contributed to the statistical analysis and the drafting of the manuscript; F. Z. contributed to the drafting of the manuscript and data analysis; C. F.-B. and N. J. T. contributed to the data collection, study design and drafting of the manuscript; A. E. and P. A. supervised the study. All the authors approved the final version of the manuscript.

None of the authors has any personal or financial conflicts of interest.

\section{References}

1. Tack J, Talley NJ, Camilleri M, et al. (2006) Functional gastroduodenal disorders. Gastroenterology 130, 1466-1479.

2. Locke GR 3rd (1998) Prevalence, incidence and natural history of dyspepsia and functional dyspepsia. Baillieres Clin Gastroenterol 12, 435-442.

3. Ford AC, Forman D, Bailey AG, et al. (2007) Initial poor quality of life and new onset of dyspepsia: results from a longitudinal 10-year follow-up study. Gut 56, 321-327.

4. Ghoshal UC, Singh R, Chang FY, et al. (2011) Epidemiology of uninvestigated and functional dyspepsia in Asia: facts and fiction. J Neurogastroenterol Motil 17, 235-244.

5. Amini E, Keshteli AH, Jazi MS, et al. (2012) Dyspepsia in Iran: SEPAHAN Systematic Review 3. Int J Prev Med 3, S18-S25.

6. Moghimi-Dehkordi B, Vahedi M, Khoshkrood Mansoori B, et al. (2011) Economic burden of gastro-oesophageal reflux disease and dyspepsia: a community-based study. Arab J Gastroenterol 12, 86-89.

7. El-Serag HB \& Talley NJ (2003) Health-related quality of life in functional dyspepsia. Aliment Pharmacol Ther 18, $387-393$

8. Moayyedi P \& Mason J (2002) Clinical and economic consequences of dyspepsia in the community. Gut 50, $10-22$.

9. Mahadeva S \& Goh KL (2006) Epidemiology of functional dyspepsia: a global perspective. World J Gastroenterol 12, 2661-2666.

10. Piessevaux H, De Winter B, Louis E, et al. (2009) Dyspeptic symptoms in the general population: a factor and cluster analysis of symptom groupings. Neurogastroenterol Motil 21, 378-388.

11. Castillo EJ, Camilleri M, Locke GR, et al. (2004) A community-based, controlled study of the epidemiology and pathophysiology of dyspepsia. Clin Gastroenterol Hepatol 2, 985-996.

12. Bisschops R, Karamanolis G, Arts J, et al. (2008) Relationship between symptoms and ingestion of a meal in functional dyspepsia. Gut 57, 1495-1503.

13. Feinle-Bisset C, Meier B, Fried M, et al. (2003) Role of cognitive factors in symptom induction following high and low fat meals in patients with functional dyspepsia. Gut 52, $1414-1418$.

14. Pilichiewicz AN, Feltrin KL, Horowitz M, et al. (2008) Functional dyspepsia is associated with a greater symptomatic response to fat but not carbohydrate, increased fasting and postprandial CCK, and diminished PYY. Am J Gastroenterol 103, 2613-2623.

15. Feinle C, Meier O, Otto B, et al. (2001) Role of duodenal lipid and cholecystokinin a receptors in the pathophysiology of functional dyspepsia. Gut 48, 347-355.

16. Barbera R, Feinle C \& Read NW (1995) Nutrient-specific modulation of gastric mechano-sensitivity in patients with functional dyspepsia. Dig Dis Sci 40, 1636-1641. 
17. Carvalho RV, Lorena SL, Almeida JR, et al. (2010) Food intolerance, diet composition, and eating patterns in functional dyspepsia patients. Dig Dis Sci 55, 60-65.

18. Kearney J, Kennedy NP, Keeling PW, et al. (1989) Dietary intakes and adipose tissue levels of linoleic acid in peptic ulcer disease. Br J Nutr 62, 699-706.

19. Saito YA, Locke GR 3rd, Weaver AL, et al. (2005) Diet and functional gastrointestinal disorders: a population-based case-control study. Am J Gastroenterol 100, 2743-2748.

20. Filipovic BF, Randjelovic T, Kovacevic N, et al. (2011) Laboratory parameters and nutritional status in patients with functional dyspepsia. Eur I Intern Med 22, 300-304.

21. Reshetnikov OV \& Kurilovich SA (2007) Mode of dieting and dyspepsia: a population-based study. Vopr Pitan 76, 35-37, (In Russian)

22. Sinn DH, Shin DH, Lim SW, et al. (2010) The speed of eating and functional dyspepsia in young women. Gut Liver $\mathbf{4}$, 173-178.

23. Pilichiewicz AN, Horowitz M, Holtmann GJ, et al. (2009) Relationship between symptoms and dietary patterns in patients with functional dyspepsia. Clin Gastroenterol Hepatol 7, 317-322.

24. Cupersus P, Keeling PW \& Gibney MJ (1996) Eating patterns in functional dyspepsia: a case control study. Eur J Clin Nutr 50, 520-523.

25. Adibi P, Keshteli AH, Esmaillzadeh A, et al. (2012) The Study on the Epidemiology of Psychological, Alimentary Health and Nutrition (SEPAHAN): overview of methodology. J Res Med Sci 17, S292-S298.
26. Sorouri M, Pourhoseingholi MA, Vahedi M, et al. (2010) Functional bowel disorders in Iranian population using Rome III criteria. Saudi J Gastroenterol 16, 154-160.

27. Hagenaars JA and Mccutcheon AL (editors) (2002) Applied Latent Class Analysis Models. Cambridge: Cambridge University Press.

28. Cameron AJ, Lagergren J, Henriksson C, et al. (2002) Gastroesophageal reflux disease in monozygotic and dizygotic twins. Gastroenterology 122, 55-59.

29. Feinle-Bisset C \& Azpiroz F (2013) Dietary and lifestyle factors in functional dyspepsia. Nature Rev Gastroenterol Hepatol 10, 150-157.

30. Feinle-Bisset C \& Horowitz M (2006) Dietary factors in functional dyspepsia. Neurogastroenterol Motil 18, 608-618.

31. Miwa H (2012) Life style in persons with functional gastrointestinal disorders - large-scale internet survey of lifestyle in Japan. Neurogastroenterol Motil 24, 464-471.

32. Nakada K, Kawasaki N, Kosone M, et al. (2006) Investigation of the eating habits of functional dyspepsia (FD) patients. Japanese Forum for Study of the Stomach 38, 18-22 (In Japanese).

33. Dynesen AW, Haraldsdottir J, Holm L, et al. (2003) Sociodemographic differences in dietary habits described by food frequency questions - results from Denmark. Eur $J$ Clin Nutr 57, 1586-1597.

34. Olinto MTA, Willett WC, Gigante DP, et al. (2011) Sociodemographic and lifestyle characteristics in relation to dietary patterns among young Brazilian adults. Public Health Nutr 14, 150-159. 